\title{
@EAD
}

REVISTA CIENTÍFICA EM EDUCAÇÃO A DISTÂNCIA

\section{EAD E SAÚDE: APROXIMAÇÃO ENTRE AS ÁREAS A PARTIR DA EXPERIÊNCIA DE UM CURSO NA FUNDAÇÃO OSWALDO CRUZ}

Maria Angélica Costa | macnf1964@yahoo.com.br

Tecnologista em Saúde Pública EAD/ENSP/FIOCRUZ, Mestre em Ciências-IOC/FIOCRUZ.

Nilton Balhis dos Santos | niltonbdossantos@gmail.com

Coordenador do Núcleo de Experimentação de Tecnologias Interativas (NEXT) do Instituto de Comunicação e Informação Científica e Tecnológica em Saúde - Fundação Oswaldo Cruz, Professor Permanente no PPGICS. Doutor em Ciência da Informação - IBICT / UFRJ

José Alberto de Francisco Rodriguez | defrancisco@gmail.com

Pesquisador da Fundação Oswaldo Cruz, Doutor em Economia da Informação pela Universidade Complutense de Madri, Espanha.

David Soeiro Barbosa | davidsoeiro@gmail.com

Pesquisador/colaborador do NEXT/ICICT/FIOCRUZ, Doutorando em Epidemiologia em Saúde Pública - ENSP/FIOCRUZ.

Thiago Petra da Silva | thiagopetra.midia@gmail.com

Pesquisador do Núcleo de Experimentação de Tecnologias Interativas da Fundação Oswaldo Cruz.

Graduado em Estudos de Mídia/UFF e Jornalismo pela UNESA.

Maria João Spilker | etutora@googlemail.com

Consultora na Fundação Oswaldo Cruz, graduada em Economia de Gestão pelo

Johann-Wolfgang Goethe Universitãt Frankfurt / Main, Alemanha.

Silvia Maria Magalhães Costa $\mid$ silmag.costa@gmail.com

Pesquisadora do Centro de Estudo e Pesquisa do Envelhecimento (CEPE)/Instituto Vital Brazil, Especialista em Educação UFRJ, em Comunicação e Saúde pelo Instituto de Comunicação e Informação Científica e Tecnológica em Saúde Fundação Oswaldo Cruz.

\section{Resumo}

Na Educação permanente em Saúde, a Educação a Distância (EAD) representa um caminho tanto para a construção coletiva e dialógica do conhecimento quanto para a melhoria das práticas dos trabalhadores do setor. A EAD na Saúde contribui também para a consolidação de um modelo mais equitativo e democrático de promoção e atenção à saúde no País. Por ser a formação e qualificação dos trabalhadores da saúde que atuam no SUS uma das missões da Fundação Oswaldo Cruz (Fiocruz), este artigo se propõe a realizar um exercício de aproximação entre as áreas de EAD e Educação em Saúde a partir da experiência do curso Andando nas Nuvens, promovido pela Fiocruz, por meio do Instituto de Comunicação e Informação Científica e Tecnológica em Saúde (ICICT), pelo Núcleo de Experimentação de Novas Tecnologias (NEXT). O curso foi analisado qualitativamente, mediante o Discurso do Sujeito Coletivo (DSC), em dois momentos, a saber: na avaliação pré-curso, com 45 discursos, e na avaliação final, com 26 discursos analisados. Nesses 71 discursos, percebeu-se que as sínteses dos DSCs na avaliação pré-curso giraram em torno de conhecimentos e interesses por pesquisas; na avaliação final do curso, as sínteses dos DSCs giraram em torno da presença ativa dos tutores e das dificuldades em lidar com as tecnologias.

\section{Palavras-chave}

EAD. Saúde. Formação. Qualificação. 
EAD AND HEALTH: APPROACH BETWEEN THESE AREAS, FROM THE EXPERIENCE OF A COURSE IN FOUNDATION OSWALDO CRUZ

\section{Abstract}

In continuing health education, EAD represents a way for both the collective and dialogical construction of knowledge as well to improve the practices of health workers. Distance education in health also contributes to the consolidation of a more equitable and democratic promotion and health care in the country. As the training and qualification of health workers who have been working in the National Health System (SUS) is one of the tasks of Foundation Oswaldo Cruz. This article proposes to carry out an exercise to bring together the areas of distance education in health education from the experience of the course Walking in the Clouds organized by the Foundation Oswaldo Cruz, through the Institute of Communication and Information Science and Technology in Health (ICICT), and the Center for Testing of New Technologies (NEXT). The course was analyzed qualitatively using the Collective Subject Discourse (DSC), on the two occasions, namely in the pre-course evaluation with 45 speeches and in final evaluation with 26 speeches analyzed. We analyzed 71 speeches, it was noted that the syntheses of DSCs in the pre-course revolved around knowledge and interests in research and in the final evaluation of the course, the syntheses of DSCs centered on the active presence of the tutors and the difficulties in dealing with the technologies.

\section{Keywords:}

EAD. Health. Training. Qualifying.

\section{Considerações iniciais}

A percepção desfavorável da sociedade civil acerca da qualidade da atenção à saúde no Brasil é sabida. As longas filas culminando com os casos de morte daqueles que buscam a assistência pública à saúde revelam um dos mais graves problemas do setor: a falta de acesso, aliada a outros tantos problemas frequentemente veiculados pela mídia.

Pensar em razões que justifiquem esse cenário não é tarefa fácil, uma vez que implicaria a análise de diversos fatores de diferentes ordens. De modo mais diretamente implicado com o objetivo deste artigo, mencionaríamos que a qualidade da atenção à saúde no Brasil pode estar relacionada com a formação e a qualificação dos trabalhadores da saúde que atuam em diversas instituições de saúde no Sistema Único de Saúde (SUS).

A formação e a qualificação desses trabalhadores da saúde é uma das missões da Fundação Oswaldo Cruz e, por essa razão, este artigo se propõe a realizar um exercício de aproximação das áreas de Educação a Distância e de Saúde a partir da experiência do curso Andando nas Nuvens: Introdução ao Uso das Tecnologias Interativas na Educação, Pesquisa, Gestão e Saúde e Noções de Computação em Nuvem, promovido pela Fiocruz, por meio do Instituto de Comunicação e Informação Científica e Tecnológica em Saúde - ICICT, através do Núcleo de Experimentação de Novas Tecnologias - NEXT.

A EAD como prática educativa e como estratégia de inovação na formação e qualificação dos trabalhadores da saúde que atuam no SUS tem na Educação - e mais especificamente na Educação permanente em Saúde - um caminho para a construção coletiva e dialógica do conhecimento, para as melhorias das práticas no corpo profissional do setor e para contribuir com a consolidação de um modelo de promoção e atenção à saúde no País mais equitativo e democrático.

O conceito de saúde conformado pelos princípios organizativos do sistema de saúde brasileiro transcende os parâmetros puramente biomédicos e assistencialistas focados na doença para a ampliação do conceito de saúde como qualidade de vida.

Esse conceito ampliado aproxima-se da Educa- 
ção, principalmente da educação permanente em Saúde para o enfrentamento da urgência de formação e qualificação dos trabalhadores do setor saúde e para o desenvolvimento de habilidades e competências consonantes com esse conceito ampliado sobre saúde.

Assim sendo, busca-se um modelo educativo/ formativo fundamentado em pressupostos teórico-conceituais direcionado para as realidades sociais, assim como para o domínio da práxis.

Entende-se que os trabalhadores da saúde não estão restritos somente aos de formação biomédica, mas também a engenheiros, arquitetos, administradores, educadores, designers e advogados, dentre outros.

Intervindo nesse âmbito de necessidades de formação dos trabalhadores da saúde, o Ministério da Saúde passou a adotar a Educação a Distância (EAD) como prática educativa estratégica capaz de possibilitar o acesso a aproximadamente 2,5 milhões de profissionais distribuídos nos mais de 5.000 municípios brasileiros, cada qual com realidades específicas, além de contextos pessoais, institucionais e profissionais heterogêneos.

\section{Contextualizando conceitos e definições na Educação a Distância}

A EAD é aqui entendida, em consonância com Moran (1994), como um processo dinâmico de ensino-aprendizagem mediado por tecnologias de informação e comunicação, em que professores/ tutores e alunos estão separados espacial e/ou temporalmente.

Preti (1996) aponta alguns aspectos a serem considerados por seus praticantes ao idealizar e elaborar cursos nesta modalidade: participantes do curso, viabilidade econômica e significância social, perfil dos candidatos, princípios de abordagem $e$ indicação de elementos curriculares.

Em relação aos princípios de abordagem, o autor aponta que os cursos a serem propostos para desenvolvimento da formação do profissional devem considerar a dimensão epistemológica no desenvolvimento do pensamento científico, pois o profissional pode obter um esquema paradigmático, de acordo com sua maneira de ver, decifrar e analisar a realidade na qual se insere e onde sua ação se constitui e é constituída por ela.

Palloff e Pratt (1999) afirmam que a chave para o processo de aprendizado a distância são as colaborações construídas a partir das interações entre os próprios estudantes e entres estes e as instituições de ensino superior e os estudantes.

Os autores indicam que no ambiente on-line o instrutor/tutor tem o papel de facilitador educacional no processo de ensino/aprendizagem na sala de aula eletrônica. Esse papel está relacionado ao modelo pedagógico de atuação do profissional na conversão da sala de aula tradicional no espaço cibernético da sala de aula eletrônica.

$\mathrm{Na}$ EAD identifica-se uma prática educativa com formas particulares de criar espaços para gerar, promover e fomentar situações favoráveis de ensino/aprendizagem entre docentes e discentes. Seu traço marcante é a mediatização das relações (LITWIN, 2001).

Belloni (2003) assinala que as definições de EAD são de modo geral descritivas, ou seja, de acordo com a perspectiva tradicional de ensino em sala de aula. Moore e Kearsley (2008) consideram que a ideia básica da EAD é simples. Nela, alunos e professores estão em locais diferentes durante todo ou em uma parte do período em que aprendem e ensinam. Ambos necessitam de algum tipo de tecnologia para a troca de informações e interação.

\section{A Educação a Distância em Saúde}

A EAD constitui uma das condições essenciais para a consolidação da reforma do setor saúde, mediante a formação e a educação permanente da força de trabalho em saúde, que deve estar preparada para enfrentar mudanças e acompanhar o acelerado avanço científico e tecnológico da sociedade contemporânea (STRUCHINER; ROSCHKE; RICCIARDI, 2002).

As autoras ressaltam a necessidade de vencer os desafios da Educação permanente em Saúde, 
compartilhando e tornando acessível o conhecimento científico produzido na solução de problemas do setor. Sendo assim, um programa de Educação permanente a Distância em Saúde com o uso de redes pode ser um novo paradigma.

Esta proposta se configura através da tecnologia educacional em saúde na busca de novas alternativas para a efetivação do programa proposto, incorporando avanços tecnológicos e científicos de diferentes áreas do conhecimento que possam oferecer novas perspectivas e novos espaços para a formação dos trabalhadores da saúde (STRUCHINER; ROSCHKE; RICCIARDI, 2002).

Esse movimento da EAD no setor saúde vai ao encontro do pressuposto político adotado pelo Ministério da Saúde, que tem na Educação permanente em Saúde (BRASIL, 2007) meios de transformar as práticas laborais por meio de novos modelos de formação de políticas, atenção, gestão, participação popular e controle social no setor saúde (OLIVEIRA, 2007).

Entende-se então, de acordo com a autora, que a educação permanente pode ser realizada via EAD como estratégia para formar e qualificar profissionais de saúde, proporcionando competência e qualidade no atendimento às pessoas que utilizam o SUS.

\section{O universo da pesquisa}

O curso Andando nas Nuvens: introdução ao uso das tecnologias interativas na educação, pesquisa, gestão e saúde e noções de computação em nuvem é uma iniciativa do Grupo de Pesquisa Tecnologias, Culturas, Práticas Interativas e Inovação em Saúde do NEXT/ICICT da Fundação Oswaldo Cruz - Fiocruz.

O objetivo do curso foi estudar, criar e implementar metodologias inovadoras que possam vir a ser utilizadas pela Incubadora de EAD no NEXT. É uma proposta de ensino a distância baseada nas necessidades que a internet pode suprir nas atividades dos trabalhadores da saúde. São elas: permitir melhor comunicação entre os trabalhadores; permitir melhor comunicação com a sociedade; permitir acesso a informação atualizada constantemente; ser ambiente de colaboração entre os trabalhadores da saúde e entre estes e a sociedade; e fornecer um ambiente contínuo de aprendizado.

Baseados nessas necessidades, os três eixos de conteúdos abordados no curso foram: colaboração, comunicação, busca e gestão da informação na internet. Com o objetivo de fazer da Internet um ambiente de aprendizado contínuo, a prioridade estava focada nas próprias experiências dos participantes desenvolvidas no curso, e não em conteúdos transmitidos.

As razões para implementar esse curso sem o auxílio de uma ferramenta específica ou de um ambiente virtual de aprendizagem (doravante denominado AVA) específico foram as seguintes: as ferramentas utilizadas para suprir o AVA são alguns dos conteúdos próprios do curso; um AVA cria uma barreira entre os alunos (ficam de um lado) e os tutores (ficam do outro lado).

O desejo era superar essa dicotomia por meio da utilização de ferramentas substitutivas que permitissem aos participantes do curso sentirem-se todos "do mesmo lado". Um AVA é um ambiente fechado, que separa os usuários do curso do resto dos internautas. A utilização de outras ferramentas abriu o curso à participação de outras pessoas, como no caso do grupo Andando nas Nuvens no Facebook, ainda ativo.

Um AVA é um ambiente organizado e coerente, e a Internet não é assim; ela é não organizada e não é coerente do ponto de vista de um AVA. Por isso, utilizar um AVA para falar de internet pode transmitir um conceito de não conformidade com a realidade da internet. Um curso em um AVA tem período de "caducidade", ou seja, depois de determinado período os participantes não podem mais acessar os conteúdos do curso nem interagir com participantes naquele ambiente.

Por outro lado, um curso sem AVA pode continuar no tempo de modo espontâneo. As ferramentas e os conteúdos ficam à disposição dos participantes, $e$ as relações criadas entre eles independem 
do AVA.

A internet não é só uma ferramenta de comunicação, uma tecnologia ou uma nova mídia; a internet é também um modelo de realidade. Como não foi usado um AVA no curso Andando nas $\mathrm{Nu}$ vens, e sim a internet como ferramenta de computação em nuvem, considera-se que o processo ensino/aprendizagem construído e vivenciado pelos participantes do curso nessa realidade é equivalente ao ensino prático.

Por não existir um AVA, pode ser necessário um tempo maior para que os alunos conheçam diversas ferramentas, o que pode não ser possível em cursos de curta duração, como cursos de atualização e aperfeiçoamento. Porém é possível realizar um curso sem AVA com auxílio de diversas ferramentas interativas, mesmo que isso demande esforço maior para tutores, orientadores de aprendizagem e coordenadores de curso.

A carga horária total do curso foi de 76 horas; 60 horas foram desenvolvidas a distância, via internet, e 16 horas foram desenvolvidas em duas atividades presenciais de oito horas cada, uma realizada no dia 24 de maio de 2011 e outra realizada no dia 04 de julho de 2011.

Foram oferecidas 30 vagas, e o contexto dos interessados em participar do curso foi o seguinte: profissionais graduados em qualquer área de atuação, que trabalham preferencialmente em instituições da área da saúde, interessados ou já inseridos em atividades/projetos que usam tecnologias interativas de informação e comunicação em processos de educação.

O projeto pedagógico do curso justificou-se pela necessidade de criação de uma rede colaborativa de caráter nacional e internacional na área da saúde que venha a fortalecer a participação do usuário na definição e adequação das políticas $e$ práticas de saúde, com ações no âmbito do SUS.

O curso foi elaborado com utilização de ferramentas de comunicação, informação e colaboração na internet, usando a web 2.0 e a computação de nuvem, percebida neste trabalho como a capacidade de armazenamento de computadores e servidores compartilhados $e$ interligados por meio da internet. Teve duração de seis semanas e houve exigência de disponibilidade de tempo para participação e dedicação aos estudos em torno de seis horas semanais.

\section{Percurso metodológico}

O curso foi fundamentado pelo princípio do trabalho colaborativo, mobilizando os participantes para a atuação coletiva propiciada pelos ambientes escolhidos para o desenvolvimento do processo ensino/aprendizagem com uma abordagem construcionista.

Segundo essa abordagem, o foco está na construção que as pessoas elaboram coletivamente (ARENDT, 2003). As interações sociais mediadas pelas tecnologias interativas possibilitaram a construção coletiva de conhecimentos entre os participantes do curso Andando nas Nuvens.

Para dar conta dessas interações sociais, optou-se pela análise dos discursos dos participantes presentes no questionário pré-curso e na avaliação final do curso, tendo por base as figuras metodológicas que constituem o discurso do sujeito coletivo (doravante denominado DSC).

Essas figuras são formadas por expressões-chave, ideias centrais, ancoragem e a síntese das figuras, denominadas discurso do sujeito coletivo.

Essa estratégia metodológica de análise privilegia os pensamentos, crenças, opiniões, percepções, valores etc. por meio da matéria discursiva presente em determinado texto, obtidos por meio de artigos de jornais, depoimentos, matérias de revistas, cartas, revistas especializadas e outros materiais textuais (LEFÈVRE; LEFÈVRE, 2003). As figuras metodológicas que compõem o DSC são as seguintes:

- Expressões-chave (doravante denominadas ECH) são pedaços, fragmentos, trechos ou transcrições literais do discurso que compõem a totalidade de um texto. A literalidade do depoimento é fundamental para que o leitor analise as condições do contexto em que foi escrito. 
- A ideia central (doravante denominada IC) é uma denominação ou expressão linguística que revela e descreve de maneira sucinta o sentido de cada discurso textual. Ela deve ser coerente com o texto em geral, e não apenas com a expressão-chave que foi selecionada. Cabe ressaltar que em uma única resposta pode haver mais de uma ideia central; por isso, o número de respostas não é igual ao número de ideias centrais identificadas no discurso.

- A ancoragem (doravante denominada AC) é a manifestação linguística que expressa explicitamente a teoria, crença, ideologia que o autor do discurso aponta em sua escrita e que está sendo utilizada para determinar uma situação específica.

Após a identificação, seleção e transcrição das expressões-chave (ECH), ideias centrais (IC) e/ou ancoragens (AC), elabora-se o discurso do sujeito coletivo (DSC), que é a síntese das figuras metodológicas citadas.

Os discursos não se anulam ou se reduzem a uma determinada categoria aglutinadora; ao contrário, são realizados quantos discursos forem necessários para expressar um determinado contexto sobre um determinado fenômeno.

As figuras metodológicas que compõem o DSC como estratégia metodológica visam o resgate do "discurso como signo de conhecimento dos próprios discursos" (LEFÉVRE; LEFÉVRE, 2003, p. 19).

\section{Análise e discussão do corpus}

Em acordo com o objetivo deste trabalho, que é realizar um exercício de aproximação das áreas de educação a distância e da saúde a partir da experiência do curso Andando nas Nuvens: introdução ao uso das tecnologias interativas na educação, pesquisa, gestão e saúde e noções de computação em nuvem, foi elaborada a análise do DSC dos discursos de participantes do curso.

Esta análise foi realizada por meio de duas questões pertinentes ao curso, realizadas em momentos diferentes: uma antes de o curso iniciar e outra ao término do curso. Poder-se-ia dizer: avaliação pré e pós-curso. Na avaliação pré-curso foram analisadas 45 respostas. Na avaliação final do curso foram analisadas 26 respostas.

\subsection{Avaliação pré-curso}

$\mathrm{Na}$ avaliação pré-curso, a questão para o entendimento do contexto e primeira aproximação entre os participantes tutores e coordenação foi a seguinte:

Qual é a sua motivação para fazer o curso Andando nas Nuvens?

IC A: Conhecimento (adquirir, ampliar, aprimorar, socializar, aprofundar, construir, potencializar).

- "Procuro novos conhecimentos, novas formas de socializar o conhecimento no processo ensino/ aprendizagem..."

Nesta ideia central a palavra de ordem é conhecimento, pois as respostas sobre a motivação em fazer o curso giraram em torno desta palavra, originando assim a ideia central A (primeira a ser analisada). Cabe ressaltar que a palavra conhecimento vem acompanhada de verbos de ação associados ao argumento de socializar o conhecimento no contexto do curso. Esta ideia-chave foi identificada em 12 discursos, o que equivale a $26,66 \%$ do total dos 45 discursos elaborados na avaliação pré-curso. Na síntese do DSC identificada por esta ideia central pode-se perceber que há a necessidade de conhecimento por parte dos trabalhadores da saúde que estavam motivados a realizarem o curso.

Essa necessidade converge com a proposta de um programa de educação permanente a distância em saúde com uso de redes, que pode ser um novo paradigma (STRUCHINER; ROSCHKE; RICCIARDI, 2002).

\section{IC B: Tecnologias interativas}

- "Tenho interesse em novas tecnologias, seja em informação/comunicação, no sentido de pes- 
quisar assuntos relacionados ao controle social, preparando-me para uma nova educação, que possa dar melhorias ao nosso Sistema Único de Saúde".

Nesta ideia central o conhecimento e a utilização de tecnologias interativas foram apontados nos discursos como motivação na realização do curso. Esta ideia central aproxima-se da proposta pedagógica do curso no que concerne à sua elaboração com a utilização de ferramentas de comunicação, informação e colaboração na internet e com a utilização da web 2.0 e da computação de nuvem.

Essa ideia central foi identificada em 11 discursos, o que equivale a $24,44 \%$ do total dos 45 discursos na avaliação pré-curso. Na síntese do DSC identificada por essa ideia central há a necessidade de conhecimento das tecnologias interativas por parte dos trabalhadores da saúde para o aprimoramento de seu cotidiano de trabalho.

\section{IC C: Interesse em pesquisas}

- "Meu interesse é de pesquisa profissional... Além disso, e talvez principalmente, ainda não achei um caminho de pesquisa que se associe com a Web 2.0, embora eu acredite que este seja um caminho importante para trilhar".

Nessa ideia central o interesse por pesquisas profissionais, acadêmicas, em redes sociais e de saúde foram explicitadas pelos respondentes. As pesquisas profissionais e acadêmicas estão muito próximas nos discursos proferidos, assim como as pesquisas em redes sociais voltadas para o controle social e para a saúde.

Essa ideia central foi identificada em 12 discursos, o que equivale a $26,66 \%$ do total dos 45 discursos na análise da avaliação pré-curso. A síntese do DSC presente nesta ideia central possibilita a argumentação de que o interesse por pesquisas profissionais e acadêmicas relacionadas a saúde, bem como por pesquisas em redes sociais, é uma demanda dos participantes que responderam à avaliação pré-curso.

\section{IC D: Formação e atuação na área da saúde}

- "Por outro lado, espero, como resultado paralelo, obter também mais orientação para atuar melhor na oferta de um curso consistente que permita minimizar o problema da falta de letramento digital na área da saúde. Nesse setor há muita resistência, em diversos níveis, muita desorientação, e o motivo principal disso é a falta de informação básica. Espero que a metodologia deste curso $e$ os conteúdos abordados possam me ajudar a atuar melhor na formação de profissionais de saúde, que tanto necessitam das novas tecnologias para atuar num mundo digital".

Nessa ideia central a emergência da formação e qualificação dos trabalhadores da saúde é explícita. Há também as questões do letramento $e$ exclusão digital na área da saúde, que são preocupações constantes dos profissionais de saúde que trabalham no planejamento e no desenvolvimento de cursos que utilizam a EAD como prática educativa e como estratégia para implementação da educação permanente em saúde.

Essa ideia central está presente em cinco discursos, o que equivale a $11,11 \%$ no universo dos 45 discursos analisados na avaliação pré-curso.

\section{IC E: A Fiocruz como instituição inovadora em saúde}

- "Bom, primeiro, quando eu vi o nome do curso, "Andando nas nuvens", achei o máximo, principalmente por sair de uma instituição como a Fiocruz, que tem toda uma tradição e tal, e me apresenta uma atividade tão inovadora. Depois, quando eu li sobre a ementa e os conteúdos e vi que o Nilton é o coordenador, pensei: eu preciso fazer esse curso; foi então que deu a maior vontade de fazer esse voo pelo céu junto com outras pessoas que querem utilizar as nuvens como estratégia de comunicação para a melhoria da saúde".

Essa ideia central aproxima-se de uma das missões da Instituição, que de formação e qualificação dos profissionais de saúde em acordo com os princípios e diretrizes do SUS. 
Como Instituição Inovadora em Saúde, a Fiocruz tem por base o tripé da pesquisa, ensino e extensão por meio de uma de suas estratégias, que é a da educação em saúde a distância.

Essa IC foi identificada em cinco discursos, o que equivale a $11,11 \%$ no universo dos 45 discursos analisados na avaliação pré-curso.

\subsection{Avaliação final do curso}

$\mathrm{Na}$ avaliação final do curso, 26 respostas são compostas de aspectos positivos e negativos, assim como de sugestões para as próximas edições do curso, dentre outras. As ideias centrais identificadas nos discursos são as seguintes:

\section{IC A: Presença ativa dos tutores}

- "Os tutores me deram muita força e orientação para a realização das atividades". Essa ideia central sintetiza um dos pilares da proposta pedagógica do curso, que é a construção coletiva do conhecimento com possibilidades de protagonismo por parte de todos os participantes sem o engessamento dos perfis alunos-tutores.

Também demonstra que o curso Andando nas Nuvens, ao criar essa dinâmica na relação entre alunos e tutores, com mediadores que iniciavam as tarefas, potencializou uma aprendizagem transversal, o que poderia ser limitado a uma relação verticalizante caso fosse usado um AVA padrão. Os tutores propiciaram a interação com os alunos e incentivaram ações colaborativas dos alunos, que auxiliavam os colegas em dificuldades.

Esta IC foi identificada em sete discursos, o que equivale a $30,76 \%$ no universo de 26 discursos analisados na avaliação final do curso.

\section{IC B: Recursos tecnológicos}

- "Achei o curso muito interessante e diferente pela autonomia que recebemos, ou seja, não estamos em nenhuma plataforma, como o Moodle, $e$ isso nos "forçou realmente" (no bom sentido) a utilizar e a nos mover pela web utilizando suas ferramentas e potencialidades. O curso me ajudou a achar, conhecer e utilizar ferramentas para meu benefício próprio, o que certamente irá agregar valor ao meu trabalho (valeu muito a pena!)."

Os mais diversos recursos tecnológicos utilizados no curso propiciou aos participantes a navegação pela web com a computação em nuvem sem um AVA específico. A ousadia dessa forma de desenvolvimento de curso pode incentivar e motivar profissionais da saúde que elaboram cursos em EAD. Os alunos teriam mais oportunidades de conhecer e se apropriar dos recursos tecnológicos e os tutores poderiam interagir muito mais com os alunos, com as ferramentas de tecnologias interativas e com os conteúdos do curso.

Esta IC está relacionada à anterior pela possibilidade da presença ativa dos tutores na interação com os recursos das tecnologias interativas apresentadas aos alunos e apropriadas por eles. Foi identificada em seis discursos, o que equivale a $23,07 \%$ do universo de 26 discursos analisados na avaliação final do curso.

\section{IC C: Participação coletiva e trabalho integrado}

- "Gostei muito da participação coletiva, todos se ajudaram [...]. Ficou bem evidenciada a importância da participação e do trabalho integrado."

Esta ideia central reforça as outras ideias centrais sobre a necessidade de os tutores estarem presentes e ativos em curso em EAD na área da saúde com a variedade de recursos tecnológicos e com a ousadia de sua criação sem um AVA específico.

A abordagem construcionista pressupõe a construção coletiva do conhecimento por meio da colaboração, da participação coletiva, e não individual. O que é possível perceber nesta IC é que essa construção envolve atividades integradas entre os participantes do curso, sejam tutores, alunos, coordenadores.

Esta IC foi identificada em sete discursos, o que equivale a $26,92 \%$ do universo de 26 discursos analisados na avaliação final do curso. 


\section{IC D: Dificuldades em lidar com as tecnologias}

- "Minha dificuldade foi administrar melhor a "enxurrada" de e-mails mesmo com os filtros (...) chega um ponto em que fica meio confuso, chato, repetitivo e com perguntas (pelos alunos) bem básicas, que ficava ao cabo dos tutores responder e interagir..."

Esta ideia central apontou a fragilidade de utilizar as ferramentas e recursos das tecnologias interativas sem discussão e avaliação prévias dos usos e limitações de determinada ferramenta.

Os alunos tinham dificuldades em ativar filtros e/ou bloquear mensagens que chegaram a suas caixas de e-mail e não lhes interessavam. Por outro lado, houve a orientação dos tutores na solução desse problema, mas ainda assim houve dificuldades, por parte dos alunos, para selecionar somente as mensagens que lhes fossem interessantes.

Esta IC foi identificada em nove discursos, o que equivale a $34,61 \%$ do universo de 26 discursos analisados na avaliação final do curso.

\section{IC E: Prazo exíguo do curso}

- "Acredito que seria bom um pouco mais de tempo para o curso, para que o cursista tenha mais tempo para as atividades."

O prazo do curso é de seis semanas, e isto pareceu aos participantes um prazo muito curto, pois eles apontaram a necessidade de mais tempo para realizar as atividades que envolviam o entendimento e a utilização de ferramentas de tecnologias interativas.

Também traz a demanda dos alunos por um curso com maior tempo de duração e talvez até em outro nível, como um curso de especialização, que teria prazo de duração maior. Numa próxima edição do curso, a possibilidade de prazo maior será analisada. Esta IC foi identificada em seis discursos, o que equivale a $23,07 \%$ no universo de 26 discursos analisados na avaliação final de curso.

\section{IC F: Dificuldades de acesso e ausência de} ferramentas utilizando computadores do setor público

- "O problema que tive foi a política de internet da instituição em que trabalho, pois bloqueou quase tudo. Assim, precisei usar o meu computador à noite para explorar os temas e executá-los."

Esta IC foi identificada pela dificuldade relatada por participantes do curso que acessavam o curso e realizam atividades do curso no local de trabalho. Em instituições públicas, por vezes é proibido ao trabalhador baixar programas, aplicativos $e$ utilizar ferramentas interativas que não sejam de interesse da instituição.

Também houve dificuldades de acesso por parte dos alunos pela ausência de ferramentas interativas. Daí a dificuldade dos quatro participantes para a utilização das ferramentas com o uso do computador em seu local de trabalho. No universo dos 26 discursos analisados, esses quatro discursos representaram 15,38\%.

\section{Considerações finais}

Em relação ao setor saúde, pode-se considerar que a EAD é uma prática educativa e uma estratégia eficaz no enfrentamento dos desafios que se fazem presentes no cotidiano de trabalho dos profissionais de saúde.

Pela educação permanente em saúde na modalidade a distância, há uma nova perspectiva para os trabalhadores da saúde que desejam formação e qualificação; a EAD é uma tendência na área da saúde (OLIVEIRA, 2007).

Nessa perspectiva, a EAD como estratégia de formação e qualificação dos profissionais de saúde do SUS fortalece e transforma a cada dia as práticas dos profissionais, colaborando para tornar o SUS produtor de equidade social e de qualidade em saúde.

Em relação ao curso Andando nas Nuvens, pode-se considerar que ele faz parte de uma das iniciativas da Fiocruz/ICICT/NEXT para consolidação das atividades de educação a distância com caráter de experimentação de novas tecnologias 
de ensino com potencial de serem incorporadas na realização de outros cursos na modalidade a distância, dentro do processo de construção da Incubadora de $\mathrm{EAD}$ que se tem buscado implementar.

A novidade de realização de um curso como este na área da saúde e a inovação tecnológica proposta pela coordenação do curso em realizar um curso EAD sem um AVA específico podem ser positivas do ponto de vista financeiro, pois a utilização de um AVA pode ser oneroso para a instituição em que o curso for desenvolvido. Também pode ser onerosa para a instituição a manutenção do sistema em que o AVA está operando.

Departamentos e unidades da Fundação Oswaldo Cruz podem desenvolver cursos em EAD na área da saúde utilizando recursos mais amigáveis e com profissionais de informática para dar suporte aos participantes do curso com investimentos mais modestos, otimizando, assim, a demanda por formação e qualificação dos trabalhadores da saúde.
Com a experiência deste curso, a EAD como prática educativa e como estratégia de formação e qualificação de profissionais e a Saúde ficam mais próximas e com maior possibilidade de enfrentamento dos desafios que se apresentam para a emergência de formação e qualificação dos trabalhadores da saúde.

As recomendações da coordenação deste curso são que um curso sem AVA específico faculta aos seus participantes enfrentar os desafios do processo ensino/aprendizagem contínuo, pois as ferramentas de tecnologias interativas ficam à disposição.

Outra recomendação é que um curso que utiliza a ferramenta de computação da nuvem como AVA pode ser realizado em instituições que desejem compartilhar conteúdos, ferramentas interativas, trabalho colaborativo sem maiores dificuldades de aprendizagem da ferramenta e com maior economia de gastos. 


\section{Referências}

ARENDT, R. J. J. Construtivismo ou construcionismo? Contribuições deste debate para a Psicologia Social. Estudos de Psicologia. Rio de Janeiro, v. 8, n. 1, p. 5-13, 2003.

BELlONI, M. L. Educação a distância. $3^{a}$ ed. Campinas: Autores Associados, 2003. (Coleção Educação Contemporânea).

BRASIL. Ministério da Saúde/Secretaria de Gestão do Trabalho e da Educação na Saúde. Departamento de Gestão da Educação na Saúde. Política Nacional de Educação Permanente em Saúde. Brasília, 2007. Disponível em: http://portal.saude.gov.br/portal/saude/profissional/visualizar_texto. cfm?idtxt=26643\&janel $=1$. Acesso em: 14 nov. 2011.

LEFÈVRE, F; LEFÈVRE, A. M. C. Discurso do sujeito coletivo: um novo enfoque em pesquisa qualitativa. Caxias do Sul: EDUCS, 2003.

LITWIN, E. Educação a distância: temas para o debate de uma nova agenda educativa. Porto Alegre: Artmed, 2001.

MOORE, M. G.; KEARSLEY. G. Educação a Distância: uma visão integrada. Trad. Roberto Galman. São Paulo: Cencage Learning, 2008.

MORAN, J. M. O que é educação à distância. Este texto foi publicado pela primeira vez com o título "Novos caminhos do ensino à distância". Informe CEAD - Centro de Educação a Distância. SENAI, Rio de Janeiro, v. 1(5): p. 1-3, out./dez. 1994.

OLIVEIRA, M. A. N. Educação a distância como estratégia para a educação permanente em saúde: possibilidades e desafios. Rev. Bras. Enferm, Brasília, v. 60(5), p. 585-589, 2007.

PALLOFF, R. M.; PRATT, K. Building learning communities in cyberspace: effective strategies for the online classroom. Califórnia: Jossey-Bass, 1999.

PRETI, O. Educação a distância: uma prática educativa mediadora e mediatizada. In: PRETI, O. Educação a distância: inícios e indícios de um percurso. Cuiabá: Ed. Da UFMT; 1996.

STRUCHINER, M.; ROSCHKE, M. A.; RICCIARDI, R. M. V. Formação permanente, flexível e à distância pela internet: Curso de Gestão Descentralizada de Recursos Humanos em Saúde. Rev. Panam Salud Publica/Pan Am J Public Health, v. 11(3), 2002. 